\title{
BMJ Open Youth Mental Health Tracker: protocol to establish a longitudinal cohort and research database for young people attending Australian mental health services
}

\author{
Cathrin Rohleder (D), Yun Ju Christine Song, Jacob J Crouse (D) , \\ Tracey A Davenport, Frank lorfino (D) , Blake Hamilton, Natalia Zmicerevska, \\ Alissa Nichles, Joanne S Carpenter (D) , Ashleigh M Tickell (D), Chloe Wilson, \\ Shane P Cross, Adam J Guastella (D), Dagmar Koethe (D), F Markus Leweke (D), \\ Elizabeth M Scott (D), Ian B Hickie
}

To cite: Rohleder C, Song YJC Crouse JJ, et al. Youth Mental Health Tracker: protocol to establish a longitudinal cohort and research database for young people attending Australian mental health services. BMJ Open 2020;10:e035379. doi:10.1136/ bmjopen-2019-035379

- Prepublication history for this paper is available online. To view these files, please visit the journal online (http://dx.doi org/10.1136/bmjopen-2019035379).

Received 30 0ctober 2019 Revised 12 February 2020 Accepted 05 May 2020
D) Check for updates

(c) Author(s) (or their employer(s)) 2020. Re-use permitted under CC BY-NC. No commercial re-use. See rights and permissions. Published by BMJ.

Brain and Mind Centre, University of Sydney, Sydney, New South Wales, Australia

Correspondence to Dr Cathrin Rohleder cathrin.rohleder@sydney.edu.au

\section{ABSTRACT}

Introduction Mental disorders are a leading cause of long-term disability worldwide. Much of the burden of mental ill-health is mediated by early onset, comorbidities with physical health conditions and chronicity of the illnesses. This study aims to track the early period of mental disorders among young people presenting to Australian mental health services to facilitate more streamlined transdiagnostic processes, highly personalised and measurement-based care, secondary prevention and enhanced long-term outcomes.

Methods and analysis Recruitment to this large-scale, multisite, prospective, transdiagnostic, longitudinal clinical cohort study ( Youth Mental Health Tracker') will be offered to all young people between the ages of 12 and 30 years presenting to participating services with proficiency in English and no history of intellectual disability. Young people will be tracked over 3 years with standardised assessments at baseline and 3, 6, 12, 24 and 36 months. Assessments will include self-report and clinician-administered measures, covering five key domains including: (1) social and occupational function; (2) self-harm, suicidal thoughts and behaviour; (3) alcohol or other substance misuse; (4) physical health; and (5) illness type, clinical stage and trajectory. Data collection will be facilitated by the use of health information technology. The data will be used to: (1) determine prospectively the course of multidimensional functional outcomes, based on the differential impact of demographics, medication, psychological interventions and other key potentially modifiable moderator variables and (2) map pathophysiological mechanisms and clinical illness trajectories to determine transition rates of young people to more severe illness forms.

Ethics and dissemination The study has been reviewed and approved by the Human Research Ethics Committee of the Sydney Local Health District (2019/ETH00469). All data will be non-identifiable, and research findings will be disseminated through peer-reviewed journals and scientific conference presentations.
Strengths and limitations of this study

This study focusses on presentation to care, rather than diagnosis-based, recruitment to establish a comprehensive and transdiagnostic longitudinal cohort and research database of young people attending Australian mental health services.

- We aim to track up to 5000 young people (aged between 12 and 30 years) over a 3-year period.

- The use of our multidimensional outcomes framework enables a comprehensive assessment of young people as well as routine monitoring.

- The study does not yet include standardised objective measures such as biomarkers, data on brain structure and function and neuropsychological assessments.

- The study is part of a clinical trials framework evaluating the utility of our multidimensional outcomes framework as well as our pathophysiological mechanism and illness trajectory model.

\section{INTRODUCTION}

Mental disorders are a leading cause of premature death and persistent disability worldwide. ${ }^{1-4}$ In those aged $10-24$ years, neuropsychiatric disorders contribute more than any other cause to the global burden of disease..$^{5}$ In addition to the early age of onset of mental disorders, factors including their prevalence, chronicity, comorbidity with physical illness, risky alcohol or other substance use, and high suicide risk and self-harm behaviour significantly contribute to significant disability and premature mortality. ${ }^{6-15}$ Consequently, earlier identification, personalised early interventions, secondary prevention and enhanced long-term care in the 
early phases of these disorders are key priorities to reduce persistent disability and premature mortality. ${ }^{16-18}$

In order to better characterise the individual needs and enable highly personalised and measurement-based care, we have proposed the use of a multidimensional outcomes framework. ${ }^{15}$ 19-21 This framework comprises five key domains, namely:

1. Social and occupational function.

2. Self-harm, suicidal thoughts and behaviours.

3. Alcohol or other substance misuse.

4. Physical health.

5. Illness type, clinical stage and trajectory.

These domains can be assessed by using various freely accessible validated scales and standardised questionnaires. ${ }^{20}$ New health information technologies (HIT), such as the InnoWell Platform (Project Synergy, InnoWell Pty), ${ }^{22}$ can facilitate the delivery of such comprehensive assessments, as they allow clinicians to implement timeefficient self-report versions of the scales and questionnaires that can often be completed by consumers without guidance. ${ }^{20}$

The assessment and identification of individual needs in each domain may prove to be particularly valuable, as it allows clinicians to develop highly personalised care options targeting specific factors associated with illness persistence and more significant disability across disorders (eg, functional impairment, physical illnesses, risky alcohol or other substance use, and high suicide risk and self-harm behaviour). ${ }^{19} 23$

Young people presenting to mental health services commonly experience a variety of symptoms that are often less specific (eg, anxiety, high level of psychological distress, sleep problems, mood instability and variable psychosocial function) and not yet sufficiently severe to meet thresholds for assigning specific diagnostic categories. Thus, current syndrome-focused classification systems, and their matching clinical guidelines, often map poorly onto the earlier phases of mental illness. ${ }^{1824-28} \mathrm{~A}$ transdiagnostic clinical staging model has been proposed as an adjunct to formal diagnosis in order to address this problem. The clinical staging model reflects the progression of mental disorders and is based on the staging concept used in general medicine, where more advanced stages are associated with a poorer prognosis and a need for more intensive interventions with a higher risk-tobenefit ratio. $^{1829}$

A detailed description of this transdiagnostic staging model is given in references. ${ }^{18}{ }^{29}$ In brief, the staging model distinguishes five stages. Each stage is defined by a degree of functional impairment and persistence of symptoms. Importantly, clinical stages are not expected to coincide with traditional diagnostic categories. The stages cover early illness phases characterised by non-specific symptoms accompanied by mild to moderate functional impairment (stage 1a) or 'attenuated syndromes' of severe mental disorders, with moderate to severe functional impairments (stage 1b), as well as full-threshold syndromes with clear and ongoing functional impairment (stage 2), and later stages, including recurrent or persistent illnesses with marked worsening in social, educational or occupational function due to persistence or recurrence (stage 3) or severe, persistent and unremitting illnesses with clear evidence of marked functional deterioration (stage 4). The staging model takes also comorbidities into account. In stage $1 \mathrm{~b}$ cases, syndromes may be mixed in terms of their symptoms or complicated by alcohol and other substance misuse. After transition to stage 2, the syndrome may remain mixed in terms of symptoms, and not necessarily matching a single or discrete Diagnostic and Statistical Manual of Mental Disorders (DSM)-style disorder, or primary discrete syndromes may co-occur. The significant comorbidity may also include alcohol or other substance misuse, abnormal eating behaviour or other relevant psychological syndromes.

General medicine also shows that an understanding of underlying pathophysiological mechanisms is crucial for selecting optimal treatment. Identifying mood and psychotic syndromes (including anxiety, depression, bipolar disorder and psychosis) based on pathophysiology will allow clinicians to select treatment options targeting underlying causes and, thus, eventually lead to improved clinical outcomes. ${ }^{30}$

Based on the results of a cross-sectional study, ${ }^{31}$ we have proposed three underlying pathophysiological mechanisms (neurodevelopmental abnormalities, hyperarousal and circadian dysfunction), which over time influence individual illness trajectories to three different illness types, namely psychosis, anxious depression and bipolar spectrum disorders, respectively. ${ }^{18}$ 31 More precisely, the 'neurodevelopmental-psychosis illness type' is characterised by psychotic features and significant and persistent developmental difficulties, including cognitive impairments, learning difficulties, and autism spectrum disorder. This subtype is based on evidence linking neurodevelopmental abnormalities with the increased risk of developing psychotic phenomena ${ }^{32-34}$ and is in line with meta-structures proposed for the redevelopment of diagnostic classification systems. ${ }^{35} 36$ The 'hyperarousalanxious depression' illness subtype includes cases with childhood anxiety, heightened stress sensitivity and adolescent depressive syndromes. Also, cases without clear evidence for a neurodevelopmental-psychosis or circadian-bipolar spectrum illness subtype are allocated to this subtype. It is consistent with models of neural fear circuitry, prolonged stress responses and glucocorticoiddependent arousal in anxiety and unipolar mood disorders. ${ }^{37-40}$ The 'circadian-bipolar spectrum' illness subtype is derived from models linking mood disorders with circadian disturbances and dysregulated activation and energy and is characterised by disrupted sleep-wake behaviours and circadian rhythms, delayed sleep-waking timing and an atypical or bipolar spectrum symptom profile. ${ }^{41-44}$

Current research projects at Brain and Mind Centre (BMC) are investigating the validity and potential implementation of this approach within mental health services. ${ }^{45} 46$ 
Importantly, there is a degree of overlap between the three pathways at all stages of illness. Previously, we have shown in a similar cohort study that $27 \%$ of young people with emerging mental disorders progress across stages and between pathways throughout care. This included 13\% progressing between pathways while developing more specific and severe symptoms. ${ }^{18}$ Continuous tracking of long-term outcomes will provide detailed information on these individual trajectories and factors influencing them, and, most importantly, will allow for early identification of changing needs that require adjustments of individual interventions. Furthermore, the information on clinical illness trajectories of young people can be used to determine transition rates to more severe illness forms (eg, severe depression, bipolar or psychotic disorder) and may help to better understand which factors drive the progression of illness.

\section{Objectives of the study and conceptual framework}

Mental disorders emerge early in life and evolve dynamically over time. The longitudinal 'Youth Mental Health Tracker' study aims to better understand the complex and variable clinical course (trajectories and pathophysiological mechanisms) of mental disorders and their impacts over time by tracking long-term multidimensional outcomes in a youth mental health cohort.

Standardised multidimensional clinical information will be routinely and confidentially collected across participating services. The study involves multiple longitudinal assessments so that key illness outcomes (ie, social and occupational function, self-harm, suicidal thoughts and behaviour, alcohol or other substance misuse, physical health, and illness type, clinical stage and trajectory) can be measured and tracked over time. Importantly, this allows for the detection of treatment non-responders at an early stage of illness; that is, before extensive exposure to interventions and chronic manifestation of illness.

In summary, such standardised data collection will enable improved identification, characterisation and profiling of mental disorders in young people, thus, enabling the identification of new targets and mechanisms that can be translated into more streamlined transdiagnostic processes, the development of the next generation of highly personalised interventions and health service strategies that greatly enhance care for young people.

\section{METHODS AND ANALYSIS \\ Study design and setting}

This is a large-scale, multisite, prospective, transdiagnostic, longitudinal clinical cohort study (Youth Mental Health Tracker), with the Brain and Mind Centre (including headspace Camperdown and Early Intervention and High-Intensity Services, public health organisations) at the University of Sydney (Sydney, Australia), being the lead site for this study. Further, St Vincent's Private Hospital (USpace) (private health organisation) will be another participating site in Sydney, Australia.
Thus, this study involves both specialist (USpace) and enhanced primary-care (headspace Camperdown and Early Intervention and High-Intensity Services) youth mental health services.

For the collection and storage of routine clinical data across sites, a HIT system will facilitate the data extraction and use in a de-identified manner for research purposes.

The study is expected to start in late 2020. Participants will be tracked over 3 years with standardised assessments occurring at baseline and 3, 6, 12, 24 and 36 months.

\section{Patient and public involvement}

The HIT system (InnoWell Platform (InnoWell Pty) $)^{22}$ that will be used by sites participating in the study for selfreport assessments was developed with the patient and public involvement and has been approved as a medical device by the Australian Register of Therapeutic Goods (ID: 315030). ${ }^{47}$ Although young people were consulted during the development of the technology used to measure relevant outcomes of the study, they were not invited to comment on the study design.

\section{Study population}

This study focusses on young people seeking treatment for emergent mood and psychotic syndromes and aims to establish a comprehensive transdiagnostic, longitudinal clinical cohort. Therefore, the recruitment is based on the presentation to care and is not restricted by specific diagnostic criteria. That is, young people presenting with non-specific anxiety or depressive symptoms according to diagnostic criteria (stage 1a), attenuated syndromes (stage 1b) or full-threshold, major and discrete syndromes (stage 2+) will be included.

This diagnosis-independent recruitment is consistent with the National Institute of Mental Health recommendations to conduct more inclusive clinical research in cohorts drawn from similar standard service settings ${ }^{48}$ and facilitate translation of the findings to other youth mental healthcare settings.

However, the vast majority who presents to the participating ambulatory-care clinical services have 'internalising' disorders (anxiety, depression, mood, psychotic disorders and so on), often associated with role impairment, comorbid substance misuse and suicidal thoughts and behaviours. The proportion of persons with major 'externalising' disorders as their primary difficulty is low.

\section{Inclusion and exclusion criteria}

Participation in this study will be offered to all young people, presenting to participating youth mental health service sites that provide mental health support to young people between the ages of 12 and 30 years. Young people who do not have proficiency in the English language or have an intellectual disability (at investigator's discretion, based on standard procedures at each site) will be excluded due to inability to accurately complete study scales and questionnaires. 
Baseline assessments including demographics,

medical and physical history

Self-report questionnaires

Clinical routine assessments

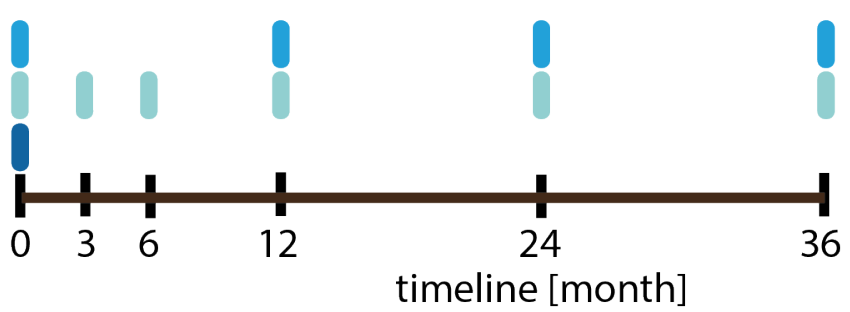

Figure 1 Overview of study visits. After completing the baseline visit, participants will be followed-up once yearly. During each visit, self-report questionnaires and clinical routine assessments have to be completed. In addition, participants will be asked to complete self-report questionnaires also 3 and 6 months after study start.

\section{Recruitment procedure}

All young people presenting to participating youth mental health services and meet inclusion and exclusion and exclusion criteria will be invited to participate in this Youth Mental Health Tracker study.

\section{Study course and procedures}

All participants recruited to this Youth Mental Health Tracker study will undergo a standardised baseline assessment ( $\mathrm{t}_{0}$, details see below), which will routinely be conducted by a mental health professional at the service. Standard information on demographics, medical history and physical history will be collected, and a range of clinician-administered assessments will be conducted. The young person will also complete a suite of online selfreport questionnaires.

Participants that complete the baseline assessment will be followed up and invited to complete an online assessment that will consist of the self-report questionnaire pack (completed online at home). These follow-up assessments will be done at 3 and 6 months following the baseline assessment $\left(\mathrm{t}_{3}, \mathrm{t}_{6}\right)$.

To ensure that optimal participant care is maintained, all participants will be invited to attend the service they initially presented to, for annual clinical routine assessments $\left(\mathrm{t}_{12}, \mathrm{t}_{24}, \mathrm{t}_{36}\right)$. Clinician administered assessments and self-report questionnaires will be repeated to track individual outcomes. That is, young people will be tracked on at least an annual basis over 36 months (figure 1).

\section{Assessments}

In order to provide improved characterisation and profiling of the Australian youth mental health population, multidimensional self-report and clinicianadministered measures (outlined below) will be deployed. These cover the five key domains of the multidimensional assessment and outcomes framework. ${ }^{15} 20$

The self-report questionnaires (see table 1) collect information regarding social and occupational function, self-harm, suicidal thoughts and behaviours, alcohol or other substance misuse, physical health as well as lifetime and current psychiatric symptoms, family history of mental illness and medical history. The questionnaires will be hosted online by using the InnoWell Platform (InnoWell Pty). ${ }^{22}$

As part of the clinical routine assessments, ${ }^{20}$ clinicians will record additional information regarding functioning, clinical stages, common illness subtypes and possible underlying pathophysiological mechanisms. More precisely this includes:

1. Social and Occupational Functioning Assessment Scale reflecting the clinician's judgement of overall social and occupational function.

2. Clinical Global Improvement, providing an overall clinician-rated summary measure that takes information on the patient's history, psychosocial circumstances, symptoms, behaviour and the impact of the symptoms in the patient's ability to function into account.

3. Common illness subtypes (psychosis, anxious depression, bipolar spectrum) and possible underlying pathophysiological mechanisms (neurodevelopmental, hyperarousal, circadian). ${ }^{1831}$

4. Clinical Staging. ${ }^{182529} 49$ Based on the clinical staging assessment, ${ }^{18} 252949$ participants will be distinguished as those in the earliest phases with non-specific clinical presentations (stages 1a 'seeking help') from those at greater-risk with more specific, sub-threshold presentations (stage 1b) or experiencing first major illness episodes (stages 2+).

Following the continuation of this Youth Mental Health Tracker study, this may further include neuropsychological and neurobiological (genetic, metabolic, circadian and imaging) assessments in a subset of participants as required by their clinicians based on need, to reflect an approach that is patient-centred care and highly personalised. 
Table 1 Overview of self-report questionnaires

\begin{tabular}{|c|c|}
\hline Health domain & Psychometric tool \\
\hline Distress & - Kessler Psychological Distress Scale-10 6263 \\
\hline $\begin{array}{l}\text { Suicidal thoughts and } \\
\text { behaviour }\end{array}$ & $\begin{array}{l}\text { The Suicidal Ideation Attributes Scale } \\
\text { - The Columbia-Suicide Severity Rating Scale } \\
\text { - }\end{array}$ \\
\hline Psychosis-like experiences & Prodromal Questionnaire-16 $6^{66}$ \\
\hline Mania-like experiences & - Altman Self-Rating Mania Scale ${ }^{67}$ \\
\hline Self-harm & - Brief Non-suicidal Self-Injury Assessment Tool ${ }^{71}$ \\
\hline Relationships & $\begin{array}{l}\text { 'Perceived social support' and 'conflict in close relationships' were measured by an adapted } \\
\text { version of the Schuster's Social Support Scale }{ }^{75}\end{array}$ \\
\hline Depression & - Quick Inventory of Depressive Symptomatology-self-report ${ }^{76}$ \\
\hline Anxiety & Overall Anxiety Severity and Impairment Scale ${ }^{77}$ \\
\hline Physical health & $\begin{array}{l}\text { Height, weight and waist circumference } \\
\text { International Physical Activity Questionnaire } \\
\text { 78 } 79\end{array}$ \\
\hline Sleep-wake cycle & $\begin{array}{l}\text { Sleep timing items are based on the Pittsburgh Sleep Quality Index }{ }^{80} \text { and Munich } \\
\text { ChronoType Questionnaire }{ }^{81} 82 \\
\text { Sleep quality items are based on expert consensus in the literature }\end{array}$ \\
\hline Post-traumatic stress & $\begin{array}{l}\text { Primary Care Posttraumatic Stess Disorder (PTSD) Screen for Diagnostic and Statistical } \\
\text { Manual of Mental Disorders Version } 5(\mathrm{DSM}-5)^{83}\end{array}$ \\
\hline
\end{tabular}

\section{Sample size calculation}

The clinics that are participating in the project provide early intervention mental health services along with assistance in promoting young peoples' well-being. As such, there is no set sample size for the establishment of this cohort. Based on the previous recruitment numbers of past research studies in these settings, the annual number of young people enrolled in the study is expected to be a minimum of 1000 . This number will sufficiently detect even the smallest effect sizes to investigate prospectively, over 3 years, the course of multidimensional functional outcomes (social and occupational function; self-harm, suicidal thoughts and behaviours; alcohol or other substance misuse and physical health) ${ }^{1519}$ in young people presenting to youth-specific mental health services.

\section{Data analysis plan}

This Youth Mental Health Tracker study will allow us to determine prospectively, over 3 years, the course of key multidimensional functional and clinical outcomes, in young people presenting to youth mental health services. This includes:
1. Modelling the impacts of demographic, treatment and other key potentially modifiable moderator variables, on functional and clinical outcomes.

2. Mapping the clinical illness trajectories and pathophysiological mechanisms of young people to determine transition rates to more severe illness forms (eg, severe depression, bipolar or psychotic disorder).

3. Investigating the differential effects of duration of exposure to antipsychotic, antidepressant, or moodstabilising medications on physical health, clinical outcomes, and risks to self-harm or suicidal behaviour.

We will make use of high-level statistical techniques, including mixed-effects/multilevel modelling, Bayesian modelling, ${ }^{50-52}$ structural equation modelling ${ }^{53}$ and data-driven techniques, ${ }^{54-56}$ such as hierarchical cluster analysis, ${ }^{57-59}$ latent profile analysis ${ }^{60}$ and group-based trajectory modelling. ${ }^{61}$

\section{ETHICS AND DISSEMINATION}

The study has been reviewed and approved by the Human Research Ethics Committee (HREC) of the Sydney Local 
Health District (2019/ETH00469, protocol version V.1-2, $01 / 07 / 2019)$. Protocol modifications will only be implemented after HREC approval.

This is a research database study; therefore, the consent process is entirely concerned with permissions regarding the storage and use of routinely collected data. For this reason, an opt-out consent process has been implemented. Potential participants presenting to the service at the participating sites will be in-depth informed by the clinicians about the study. The opt-out consent will be conducted at an 'arm's length approach'. Participants will have sufficient time to consider whether they would like to participate in the research project. Young people under the age of 15 years will initially undergo the standard consent process. However, the young participants who do not opt-out of the study will be required to obtain additional parent/guardian consent. Participants can withdraw from the study at any time. Participants will be assured that their decision to participate will not affect their treatment, nor the current or future relationship with their treating clinician or researchers at the service. All participant data will be de-identified and stored in accordance with applicable security standards; therefore, the privacy of all participants will be protected.

Research findings will be disseminated through peerreviewed journals and scientific conference presentations, and participant data will be non-identifiable.

This study allows to build a large transdiagnostic clinical cohort. The data can be used to model the clinical course and long-term functional outcomes of young people who present for clinical care before extensive exposure to interventions or chronic illness course. The study aims to improve identification, characterisation, and profiling of adolescent-onset mental disorders to enhance personalised interventions, and health service strategies that greatly enhance care for young people.

Contributors IBH conceived the research idea, designed the study and is the principal investigator. CR and YJCS contributed to study conception and wrote the study protocol with input of IBH, JC, TAD, FI, BH, NZ, AN, JSC, AMT, CW, SC, AJG, DK, FML and EMS. CR wrote the manuscript with input of IBH, YJCS, JC, TAD, FI, BH, NZ, AN, JSC and AMT. All authors critically reviewed content and approved the final version of the publication.

Funding This project is an investigator-initiated trial and will be supported by philanthropic funding, for which donor(s) who are families affected and wish to remain anonymous. IBH is supported by a National Health and Medical Research Council (NHMRC) Senior Principle Research Fellowship grant number 1136259.

Competing interests Professor lan Hickie was an inaugural Commissioner on Australia's National Mental Health Commission (2012-18). He is the Co-Director, Health and Policy at the Brain and Mind Centre (BMC) University of Sydney. The $B M C$ operates an early-intervention youth services at Camperdown under contract to headspace. He is the Chief Scientific Advisor to, and a $5 \%$ equity shareholder in, InnoWell Pty Ltd. InnoWell was formed by the University of Sydney ( $45 \%$ equity) and PwC (Australia; 45\% equity) to deliver the \$30 M Australian Government-funded Project Synergy (2017-20; a three-year program for the transformation of mental health services) and to lead transformation of mental health services internationally through the use of innovative technologies.

Patient and public involvement Patients and/or the public were not involved in the design, or conduct, or reporting, or dissemination plans of this research.

Patient consent for publication Not required.

Provenance and peer review Not commissioned; externally peer reviewed.
Open access This is an open access article distributed in accordance with the Creative Commons Attribution Non Commercial (CC BY-NC 4.0) license, which permits others to distribute, remix, adapt, build upon this work non-commercially, and license their derivative works on different terms, provided the original work is properly cited, appropriate credit is given, any changes made indicated, and the use is non-commercial. See: http://creativecommons.org/licenses/by-nc/4.0/.

\section{ORCID iDs}

Cathrin Rohleder http://orcid.org/0000-0002-3559-1846 Jacob J Crouse http://orcid.org/0000-0002-3805-2936 Frank lorfino http://orcid.org/0000-0003-1109-0972 Joanne S Carpenter http://orcid.org/0000-0002-9766-6700 Ashleigh M Tickell http://orcid.org/0000-0002-8000-7864 Adam J Guastella http://orcid.org/0000-0001-8178-4625 Dagmar Koethe http://orcid.org/0000-0002-8324-6756

F Markus Leweke http://orcid.org/0000-0002-8163-195X

Elizabeth M Scott http://orcid.org/0000-0003-3907-0324

Ian B Hickie http://orcid.org/0000-0001-8832-9895

\section{REFERENCES}

1 Whiteford HA, Degenhardt L, Rehm J, et al. Global burden of disease attributable to mental and substance use disorders: findings from the global burden of disease study 2010. Lancet 2013;382:1575-86.

2 Erskine HE, Moffitt TE, Copeland WE, et al. A heavy burden on young minds: the global burden of mental and substance use disorders in children and youth. Psychol Med 2015;45:1551-63.

3 Vigo D, Thornicroft G, Atun R. Estimating the true global burden of mental illness. Lancet Psychiatry 2016;3:171-8.

4 Walker ER, McGee RE, Druss BG. Mortality in mental disorders and global disease burden implications: a systematic review and metaanalysis. JAMA Psychiatry 2015;72:334-41.

5 Gore FM, Bloem PJN, Patton GC, et al. Global burden of disease in young people aged 10-24 years: a systematic analysis. Lancet 2011;377:2093-102.

6 Moran P, Coffey C, Romaniuk H, et al. The natural history of selfharm from adolescence to young adulthood: a population-based cohort study. Lancet 2012;379:236-43.

7 Hawton K, Saunders KEA, O'Connor RC. Self-harm and suicide in adolescents. Lancet 2012;379:2373-82.

8 De Leo D, Heller TS. Who are the kids who self-harm? an Australian self-report school survey. Med J Aust 2004;181:140-4.

9 Scott EM, Hermens DF, Glozier N, et al. Targeted primary carebased mental health services for young Australians. Med J Aust 2012;196:136-40.

10 Gustavsson A, Svensson M, Jacobi F, et al. Cost of disorders of the brain in Europe 2010. Eur Neuropsychopharmacol 2011;21:718-79.

11 Stuart MJ, Baune BT. Depression and type 2 diabetes: inflammatory mechanisms of a psychoneuroendocrine co-morbidity. Neurosci Biobehav Rev 2012;36:658-76.

12 Scott EM, Carpenter JS, lorfino F, et al. What is the prevalence, and what are the clinical correlates, of insulin resistance in young people presenting for mental health care? A cross-sectional study. BMJ Open 2019;9:e025674.

13 Merikangas KR, Mehta RL, Molnar BE, et al. Comorbidity of substance use disorders with mood and anxiety disorders: results of the international consortium in psychiatric epidemiology. Addict Behav 1998;23:893-907.

14 Hermens DF, Scott EM, White D, et al. Frequent alcohol, nicotine or cannabis use is common in young persons presenting for mental healthcare: a cross-sectional study. BMJ Open 2013;3. doi:10.1136/ bmjopen-2012-002229. [Epub ahead of print: 04 Feb 2013].

15 Iorfino F, Carpenter JS, Cross SP, et al. Multidimensional outcomes in youth mental health care: what matters and why? Med J Aust 2019;211:S4-11.

16 Lopez AD, Mathers CD, Ezzati M, et al. Global and regional burden of disease and risk factors, 2001: systematic analysis of population health data. Lancet 2006;367:1747-57.

17 Rohleder C, Crouse JJ, Carpenter JS, et al. Personalising care options in youth mental health: using multidimensional assessment, clinical stage, pathophysiological mechanisms, and individual illness trajectories to guide treatment selection. Med J Aust 2019;211:S32-41.

18 Carpenter JS, lorfino F, Cross SP, et al. Combining clinical stage and pathophysiological mechanisms to understand illness trajectories in young people with emerging mood and psychotic syndromes. Med $\mathrm{J}$ Aust 2019;211:S12-22. 
19 lorfino F, Hickie IB, Lee RSC, et al. The underlying neurobiology of key functional domains in young people with mood and anxiety disorders: a systematic review. BMC Psychiatry 2016;16:156.

20 Crouse JJ, Rohleder C, Carpenter JS, et al. A comprehensive assessment framework for youth mental health: guiding highly personalised and measurement-based care using multidimensional and objective measures. Med J Aust 2019;211:S23-31.

21 Scott EM, Carpenter JS, lorfino F, et al. Early intervention, prevention and prediction in mood disorders: tracking multidimensional outcomes in young people presenting for mental health care. In: Baune BT, ed. Personalized psychiatry. Elsevier, 2019.

22 Iorfino F, Cross SP, Davenport T, et al. A digital platform designed for youth mental health services to deliver personalized and Measurement-Based care. Front Psychiatry 2019;10:595.

23 Sahakian BJ, Malloch G, Kennard C, et al. A UK strategy for mental health and wellbeing. Lancet 2010;375:1854-5.

24 Fergusson DM, Horwood LJ, Ridder EM, et al. Subthreshold depression in adolescence and mental health outcomes in adulthood. Arch Gen Psychiatry 2005;62:66-72.

25 Hickie IB, Scott EM, Hermens DF, et al. Applying clinical staging to young people who present for mental health care. Early Interv Psychiatry 2013;7:31-43.

26 Hickie IB, Scott J, Hermens DF, et al. Clinical classification in mental health at the cross-roads: which direction next? BMC Med 2013;11:125

27 Patel V, Saxena S, Lund C, et al. The Lancet Commission on global mental health and sustainable development. Lancet 2018;392:1553-98.

28 Scott J, Henry C. Clinical staging models: from general medicine to mental disorders. BJPsych Adv 2017;23:292-9.

29 Hickie IB, Carpenter JS, lorfino F, et al. The Utility of Clinical Staging in Youth Mental Health Settings: Neurobiological and Longitudinal data from Sydney-based studies of transdiagnostic cohorts. In: McGorry P, Hickie IB, eds. Clinical staging in psychiatry: making diagnosis work for research and treatment. Cambridge University Press, 2019.

30 Insel T, Cuthbert B, Garvey M, et al. Research domain criteria (RDoC): toward a new classification framework for research on mental disorders. Am J Psychiatry 2010;167:748-51.

31 Hickie IB, Hermens DF, Naismith SL, et al. Evaluating differential developmental trajectories to adolescent-onset mood and psychotic disorders. BMC Psychiatry 2013;13:303.

32 Bombin I, Mayoral M, Castro-Fornieles J, et al. Neuropsychological evidence for abnormal neurodevelopment associated with earlyonset psychoses. Psychol Med 2013;43:757-68.

33 Peralta V, de Jalón EG, Campos MS, et al. The meaning of childhood attention-deficit hyperactivity symptoms in patients with a firstepisode of schizophrenia-spectrum psychosis. Schizophr Res 2011;126:28-35.

34 Piper M, Beneyto M, Burne THJ, et al. The neurodevelopmental hypothesis of schizophrenia: convergent clues from epidemiology and neuropathology. Psychiatr Clin North Am 2012;35:571-84.

35 Andrews G, Pine DS, Hobbs MJ, et al. Neurodevelopmental disorders: cluster 2 of the proposed meta-structure for DSM-V and ICD-11. Psychol Med 2009;39:2013-23.

36 Andrews G, Goldberg DP, Krueger RF, et al. Exploring the feasibility of a meta-structure for DSM-V and ICD-11: could it improve utility and validity? Psychol Med 2009;39:1993-2000.

37 Hansell NK, Wright MJ, Medland SE, et al. Genetic co-morbidity between neuroticism, anxiety/depression and somatic distress in a population sample of adolescent and young adult twins. Psychol Med 2012;42:1249-60.

38 Bennett A O MR. Stress and anxiety in schizophrenia and depression: glucocorticoids, corticotropin-releasing hormone and synapse regression. Aust N Z J Psychiatry 2008;42:995-1002.

39 Charney DS. Psychobiological mechanisms of resilience and vulnerability: implications for successful adaptation to extreme stress. Am J Psychiatry 2004;161:195-216.

40 McGowan PO, Sasaki A, D'Alessio AC, et al. Epigenetic regulation of the glucocorticoid receptor in human brain associates with childhood abuse. Nat Neurosci 2009;12:342-8.

41 Woo YS, Shim IH, Wang H-R, et al. A diagnosis of bipolar spectrum disorder predicts diagnostic conversion from unipolar depression to bipolar disorder: a 5-year retrospective study. J Affect Disord 2015; 174:83-8.

42 Scott J, Murray G, Henry C, et al. Activation in bipolar disorders: a systematic review. JAMA Psychiatry 2017;74:189-96.

43 Mitchell PB, Goodwin GM, Johnson GF, et al. Diagnostic guidelines for bipolar depression: a probabilistic approach. Bipolar Disord 2008;10:144-52.
44 Akiskal HS, Benazzi F. Atypical depression: a variant of bipolar II or a bridge between unipolar and bipolar II? J Affect Disord 2005;84:209-17.

45 Davenport TA, LaMonica HM, Whittle L, et al. Validation of the InnoWell platform: protocol for a clinical trial. JMIR Res Protoc 2019;8:e13955.

46 LaMonica HM, Davenport TA, Braunstein K, et al. Technologyenabled person-centered mental health services reform: strategy for implementation science. JMIR Ment Health 2019;6:e14719-e6.

47 Hickie IB, Davenport TA, Burns JM, et al. Project synergy: codesigning technology-enabled solutions for Australian mental health services reform. Med J Aust 2019;211:S3-39.

48 Cuthbert BN. The RDoC framework: facilitating transition from ICD/ DSM to dimensional approaches that integrate neuroscience and psychopathology. World Psychiatry 2014;13:28-35.

49 Hickie IB, Scott J, McGorry PD. Clinical staging for mental disorders: a new development in diagnostic practice in mental health. Med $J$ Aust 2013;198:461-2.

50 Sojo VE, Wood RE, Wood SA, et al. Reporting requirements, targets, and quotas for women in leadership. Leadersh Q 2016;27:519-36.

51 Lee RSC, Hermens DF, Scott J, et al. A transdiagnostic study of education, employment, and training outcomes in young people with mental illness. Psychol Med 2017:47:2061-70.

52 Iorfino F, Scott EM, Carpenter JS, et al. Clinical stage transitions in persons aged 12 to 25 years presenting to early intervention mental health services with anxiety, mood, and psychotic disorders. JAMA Psychiatry 2019. doi:10.1001/jamapsychiatry.2019.2360. [Epub ahead of print: 28 Aug 2019].

53 Lee RSC, Hermens DF, Naismith SL, et al. Clinical, neurocognitive and demographic factors associated with functional impairment in the Australian brain and mind youth cohort study (2008-2016). BMJ Open 2018;8:e022659.

54 Lee RSC, Hermens DF, Naismith SL, et al. Neuropsychological and functional outcomes in recent-onset major depression, bipolar disorder and schizophrenia-spectrum disorders: a longitudinal cohort study. Transl Psychiatry 2015;5:e555.

55 Hermens DF, Lagopoulos J, Naismith SL, et al. Distinct neurometabolic profiles are evident in the anterior cingulate of young people with major psychiatric disorders. Trans/ Psychiatry 2012;2:e110

56 Hermens DF, Naismith SL, Chitty KM, et al. Cluster analysis reveals abnormal hippocampal neurometabolic profiles in young people with mood disorders. Eur Neuropsychopharmacol 2015;25:836-45.

57 Tickell AM, Scott EM, Davenport T, et al. Developing neurocognitive standard clinical care: a study of young adult inpatients. Psychiatry Res 2019;276:232-8.

58 Tickell AM, Scott EM, Davenport T, et al. Neurocognitive clusters: a pilot study of young people with affective disorders in an inpatient facility. J Affect Disord 2019;242:80-6.

59 Crouse JJ, Moustafa AA, Bogaty SER, et al. Parcellating cognitive heterogeneity in early psychosis-spectrum illnesses: a cluster analysis. Schizophr Res 2018;202:91-8.

60 Crouse JJ, Chitty KM, lorfino F, et al. Exploring associations between early substance use and longitudinal socio-occupational functioning in young people engaged in a mental health service. PLoS One 2019;14:e0210877.

61 lorfino F, Hermens DF, Cross SP, et al. Delineating the trajectories of social and occupational functioning of young people attending early intervention mental health services in Australia: a longitudinal study. BMJ Open 2018;8:e020678.

62 Kessler RC, Andrews G, Colpe LJ, et al. Short screening scales to monitor population prevalences and trends in non-specific psychological distress. Psychol Med 2002;32:959-76.

63 Andrews G, Slade T. Interpreting scores on the Kessler psychological distress scale (K10). Aust N Z J Public Health 2001:25:494-7.

64 van Spijker BAJ, Batterham PJ, Calear AL, et al. The suicidal ideation attributes scale (SIDAS): community-based validation study of a new scale for the measurement of suicidal ideation. Suicide Life Threat Behav 2014;44:408-19.

65 Posner K, Brown GK, Stanley B, et al. The Columbia-Suicide severity rating scale: initial validity and internal consistency findings from three multisite studies with adolescents and adults. Am J Psychiatry 2011;168:1266-77.

66 Ising HK, Veling W, Loewy RL, et al. The validity of the 16-item version of the prodromal questionnaire $(\mathrm{PQ}-16)$ to screen for ultra high risk of developing psychosis in the general help-seeking population. Schizophr Bull 2012;38:1288-96.

67 Altman EG, Hedeker D, Peterson JL, et al. The Altman self-rating mania scale. Biol Psychiatry 1997;42:948-55.

68 Üstün TB, Kostanjesek N, Chatterji S, et al. Measuring health and disability: manual for WHO disability assessment schedule 
(WHODAS 2.0), 2010. Available: https://apps.who.int/iris/handle/ 10665/43974\%20World\%20Health\%20Organization

69 Mundt JC, Marks IM, Shear MK, et al. The work and social adjustment scale: a simple measure of impairment in functioning. $\mathrm{Br}$ J Psychiatry 2002;180:461-4.

70 Goldman HH, Skodol AE, Lave TR. Revising axis V for DSMIV: a review of measures of social functioning. Am J Psychiatry 1992;149:1148-56.

71 Whitlock J, Exner-Cortens D, Purington A. Assessment of nonsuicidal self-injury: development and initial validation of the Non-Suicidal SelfInjury-Assessment tool (NSSI-AT). Psychol Assess 2014;26:935-46.

72 Humeniuk R, Ali R, Babor TF, et al. Validation of the alcohol, smoking and substance involvement screening test (assist). Addiction 2008;103:1039-47.

73 WHO ASSIST Working Group. The alcohol, smoking and substance involvement screening test (assist): development, reliability and feasibility. Addiction 2002;97:1183-94.

74 Bush K, Kivlahan DR, McDonell MB, et al. The audit alcohol consumption questions (AUDIT-C): an effective brief screening test for problem drinking. ambulatory care quality improvement project (ACQUIP). alcohol use disorders identification test. Arch Intern Med 1998;158:1789-95.

75 Schuster TL, Kessler RC, Aseltine RH. Supportive interactions, negative interactions, and depressed mood. Am J Community Psychol 1990;18:423-38.

76 Rush AJ, Trivedi MH, Ibrahim HM, et al. The 16-Item quick inventory of depressive symptomatology (QIDS), clinician rating (QIDS-C), and self-report (QIDS-SR): a psychometric evaluation in patients with chronic major depression. Biol Psychiatry 2003;54:573-83.

77 Norman SB, Cissell SH, Means-Christensen AJ, et al. Development and validation of an overall anxiety severity and impairment scale (OASIS). Depress Anxiety 2006;23:245-9

78 Booth M. Assessment of physical activity: an international perspective. Res Q Exerc Sport 2000;71:114-20.

79 Craig CL, Marshall AL, Sjöström M, et al. International physical activity questionnaire: 12 -country reliability and validity. Med Sci Sports Exerc 2003;35:1381-95.

80 Buysse DJ, Reynolds CF, Monk TH, et al. The Pittsburgh sleep quality index: a new instrument for psychiatric practice and research. Psychiatry Res 1989;28:193-213.

81 Zavada A, Gordijn MCM, Beersma DGM, et al. Comparison of the Munich chronotype questionnaire with the horne-ostberg's morningness-eveningness score. Chronobiol Int 2005;22:267-78.

82 Roenneberg T, Wirz-Justice A, Merrow M. Life between clocks: daily temporal patterns of human chronotypes. J Biol Rhythms 2003;18:80-90.

83 Prins A, Bovin MJ, Smolenski DJ, et al. The primary care PTSD screen for DSM-5 (PC-PTSD-5): development and evaluation within a veteran primary care sample. J Gen Intern Med 2016;31:1206-11.

84 Fairburn CG, Cooper Z. The Eating Disorder Examination. In: Binge eating: nature, assessment, and treatment. 12 edn. New York, US: Guilford Press, 1993: 317-60. 